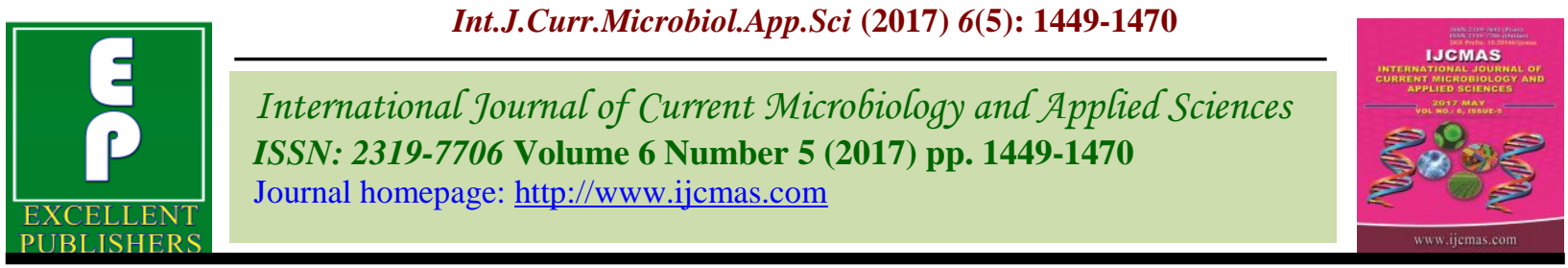

Original Research Article

https://doi.org/10.20546/ijcmas.2017.605.159

\title{
Prevalence of Extended Spectrum Beta-Lactamase Producing Klebsiella Species in an Intensive Care Unit
}

\author{
V. Sangamithra*, Shamsadh, Kalyani and Mallika \\ Department of Microbiology, SRM Medical College and RI, Chennai, India \\ *Corresponding author
}

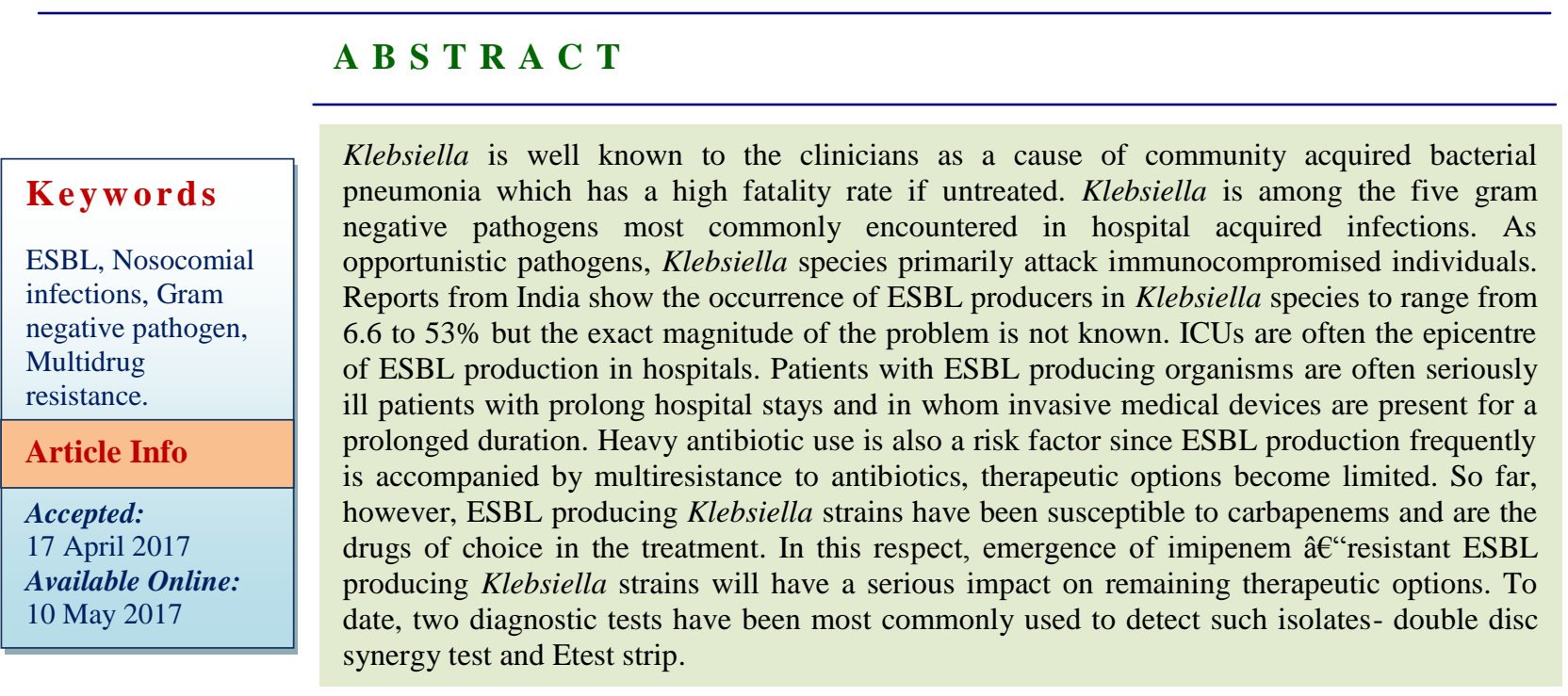

\section{Introduction}

Members of the family Enterobacteriaceae are frequently encountered in hospital acquired infection as they are more important in the spread of non enteric infection in hospital. This is due to the antibiotic resistance, transmissibility, and virulence of the organism, which interact among the patients with similar medical problems who undergo similar procedure and receive similar antibiotics. Nosocomial infections carry considerable clinical and economic burden. Klebsiella species are ubiquitous in nature. They probably have two common habitats, one being the environment, where they are found in surface water, sewage and soil and on plants and the other being the mucosal surfaces of mammals such as humans, horses or swine which they colonize (Ullmann et al., 1998). Klebsiella is well known to most clinicians as a cause of community acquired bacterial pneumonia occurring particularly in chronic alcoholics (Aggarwal et al., 2003).

In hospitals, colonization rates increase with the duration of stay and the hospital personnel can carry the organism. The high rate of colonization in patients is associated with the use of antibiotics (Patrick Grimont et al., 2005). 
As opportunistic pathogens, Klebsiella species primarily attack immunocompromised individuals who are hospitalized and suffer from severe underlying diseases like diabetes mellitus or chronic obstructive pulmonary disease (COPD) (Podschun et al., 1998).

The bowel is the major site of colonization with infection of the urinary tract, respiratory tract, and wounds. In addition to prior antibiotic use, risk factors for infection and colonization include the presence of an indwelling catheter, prolonged use of invasive medical devices, feeding tube, or central venous catheter; poor health status; severe illness, including major surgery and treatment in an intensive care unit (ICU) or nursing home, inadequate infection control practices. Acquisition of these species has become a major problem in most hospitals because of resistance to multiple antibiotics and potential transfer of plasmids to other organisms (Obiamive et al., 2002). Morbidity and mortality rates are comparable to those for other gram-negative organism causing sepsis and septic shock. In neonatal units, outbreaks caused by ESBL producing strains result in more serious problem and may be associated with increased mortality (Obiamive Umeh et al., 2002). Among Klebsiella species, Klebsiella pneumoniae can cause primary community acquired pneumonia as well as nosocomial pneumonia. The typical case is a middle or elderly male with underlying problems such as alcoholism, COPD, or diabetes mellitus. Necrosis and abscess formation is more likely with Klebsiella pneumoniae infections than with any other bacterial pneumonia. In addition to pneumonia, Klebsiella can cause urinary tract and wound infections, bacteremia, and meningitis.

Klebsiella pneumoniae rank $7^{\text {th }}$ as a cause of nosocomial UTI, blood stream, cardiovascular, and ear, nose and throat infections (Sharon Abbott et al., 2003). They rank $4^{\text {th }}$ as a cause of hospital acquired pneumonia (Sharon Abbott et al., 2003).

In contrast, infections due to Klebsiella pneumoniae subspecies ozaenae and Klebsiella pneumoniae subspecies rhinoscleromatis are restricted to certain body sites and in most cases affect only the nose (Ingo Stock et al., 2000, Sharon Abbott et al., 2003) causing atrophic rhinitis and rhinoscleroma respectively. Both are chronic diseases of the upper respiratory tract; occurring most frequently in tropical areas of the world; transmission is thought to be from person to person.

In pediatric wards, nosocomial Klebsiella infections are especially troublesome particularly in premature infants and ICUs. Klebsiella species are often the pathogens involved in neonatal sepsis in both early manifestation and late manifestation infections (Podschun and Ullmann, 1998). Klebsiella oxytoca in particular has been implicated in neonatal bacteremia, especially among premature infants and in neonatal ICUs. It is among the top 4 pathogens that cause infection in patients in neonatal intensive care units. It is the second most frequent cause of gram-negative neonatal bacteremia (Obiamive Umeh et al., 2002).

Almost universally, the members of this genus are resistant to the early beta lactam antimicrobials such as penicillin, ampicillin, and amoxicillin. They are usually susceptible to the cephalosporins which are the drugs of choice. However in recent years Klebsiella species resistant to cephalosporin are emerging rapidly. This resistance is due the presence of a group of enzymes called extended spectrum beta lactamases. The emergence of extended spectrum beta lactamases is an increasing problem worldwide and is due to indiscriminate use of 
the $3^{\text {rd }}$ generation cephalosporins. They were unknown before the introduction of these antibiotics in the early 1980s.

ESBL producing organisms are now a problem in the hospitalized patients worldwide. The ESBL phenomenon began in Western Europe, most likely because extended spectrum beta lactam antibiotics were first used there clinically; however it did not take long before ESBL had been detected in the United States and Asia (Obiamive Umeh et al., 2002).

ESBLs are enzymes that have the ability to inactivate beta lactam antibiotics containing oxyiminogroup ( $3{ }^{\text {rd }}$ generation cephalosporins and Aztreonam). Hence ESBLs are capable of hydrolyzing broad spectrum cephalosporins, penicillins, and monobactams, but are inactive against Cephamycins and Carbapenems.

The ESBL producing bacteria are typically associated with Multidrug resistance because genes for other mechanisms of resistance often reside on the same plasmid as the ESBL genes. Thus some ESBL producing strains also show resistance to Quinolones, Aminoglycosides and Trimethoprim sulfamethoxazole.

Infections with ESBL producing bacteria can result in avoidable failure of treatment with resultant increase in the cost of patient care with prolong hospital stay. ESBL producing organisms also exhibit cross resistance to various other classes of antibiotics in common use resulting in limitation of therapeutic options.

The concern for the accurate detection of ESBLs is twofold. First, there is an increasing prevalence of ESBLs worldwide. Second, many strains producing ESBLs demonstrate an inoculum effect, in that the Minimum inhibitory concentrations of extended spectrum cephalosporins rise as the inoculum increases (Patricia Bradford, 2001) Extended spectrum beta lactamases producing Klebsiella pneumoniae was first reported in 1983 from Germany. Since then the usage of $3^{\text {rd }}$ generation cephalosporin in the treatment of multidrug resistant Klebsiella pneumoniae infections has been limited as resistant strains have been reported from other parts of the world and recently from South India also (Jerestin Hansotia et al., 1997). Production of these enzymes is either chromosomally mediated or plasmid mediated. Point aminoacid substitution of the classical plasmid mediated betalactamases like TEM-1, TEM-2 and SHV-1 increases the spectrum of activity from earlier generation betalactams to $3^{\text {rd }}$ generation cephalosporins and monobactams.

The chromosomally mediated betalactamases production is mainly through the expression of AmpC gene which is either constitutive or inducible (Rodrigues et al., 2004). Klebsiella are a part of normal life and live inside almost every individual. As opportunistic pathogens, they take advantage of weakened host defenses to colonize and elicit a variety of disease states. Many hospital-acquired infections occur because of the invasive treatments that are often needed in hospitalized patients leading to an increase in the susceptibility to infection. Due to extensive spread of antibiotic resistance, especially extended spectrum betalactamase producing strains, there has been renewed interest in Klebsiella infections.

The main aim of this study includes, to isolate and speciate the Klebsiella isolates from various clinical specimens from patients admitted in ICU. To determine the antibiotic susceptibility pattern of Klebsiella species by disc diffusion method. And to detect the presence of extended spectrum betalactamases (ESBL) by double disc synergy test and Inhibitor Potentiated disc diffusion test. 


\section{Materials and Methods}

A prospective study was undertaken from November 2004 to April 2005 in the Department of Microbiology, SriRamachandra Medical College and Research Institute, a 1500-bedded tertiary care centre. During this period all clinically significant, consecutive, non repetitive isolates of the genus Klebsiella from ICU patients were included in the study. The isolates were collected from various specimens like blood, urine, pus, wound swab, sputum, bronchial wash, endotracheal secretions and body fluids from patients admitted in medical and surgical intensive care units (medical, surgical, cardiothoracic, cardiology, neurosurgery and burns units).

A detailed clinical history was taken and recorded from the patients whose culture grew Klebsiella from any of the above clinical specimens. The proforma included the patient's age, sex, date of admission, admitted ward, brief clinical history, diagnosis, presence of any risk factors (DM, intake of steroid or immunosuppressant, HIV, HBV), presence of associated illness and antibiotic therapy. The samples were collected aseptically by standard techniques (Elmer Koneman et al., 1997).

\section{Methodology}

\section{Specimen processing}

A direct smear for assessment of the cellularity and presence of bacteria was carried out in all cases. The media for the study were procured from Himedia (Mumbai).The media and the biochemicals were prepared by following standard procedures (Collee et al., 1996) (Annexure II). Each batch of media and biochemicals were tested with suitable controls and was utilized only if it was satisfactory. The primary isolation of the specimen was done on 5\% sheep blood agar, MacConkey agar and incubated overnight at $37^{\circ} \mathrm{C}$. The isolates produced large grey colonies with a mucoid consistency on blood agar and lactose fermenting large pink coloured mucoid colonies on MacConkey agar. The isolates were subjected to Gram stain which showed capsulated gram-negative short straight rods uniformly stained with parallel sides and rounded ends. A preliminary biochemical reaction which includes catalase test, oxidase tests, test for indole production, triple sugar iron (TSI) reaction, urease test, citrate utilization and mannitol motility test were performed. The Oxidative-Fermentative test for glucose was put for each isolate to show the ability of the organism to breakdown carbohydrates both aerobically and anaerobically.

\section{Biochemical reactions}

Once presumptively identified as belonging to the family Enterobacteriaceae and genus Klebsiella the organism was subjected to further identification up to species level based on Bergey's Manual. The isolates were also subjected to tests for specific breakdown products formed from fermentation of glucose, Methyl Red and Voges-Proskauer (MR/VP).

Citrate utilization test and tests for enzymes which included Urease and nitrate reduction test was performed. Finally aminoacid decarboxylation reactions were performed for amino acids lysine and ornithine, colour change was observed at the end of each day. A consequent change in colour to violet or reddish-violet was observed and considered a positive result. Based on these tests the isolates were identified as Klebsiella pneumoniae subspecies pneumoniae, Klebsiella oxytoca, Klebsiella planticola and Klebsiella pneumoniae subspecies ozaenae. 


\section{Antibiotic susceptibility testing}

Antibiotic susceptibility testing was done on Muller Hinton agar plates by Kirby Bauer disc diffusion method. ATCC, E.coli 25922 and Klebsiella pneumoniae ATCC 700603 were used as control strain and was included for each batch of antibiogram of the test strain.

The antibiotic discs namely Ampicillin (A$10 \mu \mathrm{g})$, Pipericillin (Pc-100 $\mu \mathrm{g})$, Ciprofloxacin (Cf-5 $\mu \mathrm{g}$ ), Amikacin (Ak-30 $\mu \mathrm{g}$ ), Cefazolin $(\mathrm{Cz}-30 \mu \mathrm{g})$, Cefuroxime $(\mathrm{Cu}-30 \mu \mathrm{g})$, Ceftazidime (Ca-30 $\mu$ g), Cefotaxime (Ce$30 \mu \mathrm{g})$, Ceftriaxone $(\mathrm{Ci}-30 \mu \mathrm{g})$, Cefaperazone (Cs-75 $\mu \mathrm{g})$, Cefoxitin (Cfx-30 $\mu \mathrm{g})$, Cefepime (Cpm-30 $\mu \mathrm{g})$, Ceftazidime-clavulanate (Ca$30 \mu \mathrm{g}$, Clavulanate-10 $\mu \mathrm{g}$ ), Amoxyclav Amox$20 \mu \mathrm{g}$, Clav-10 $\mu \mathrm{g}$ ) and Imipenem (I-10 $\mu \mathrm{g})$ were obtained from Himedia. Antibiotic disc

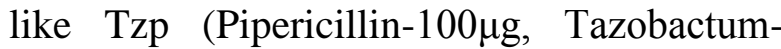
$10 \mu \mathrm{g})$ from $\mathrm{BBL}$ and Cefaperazone-

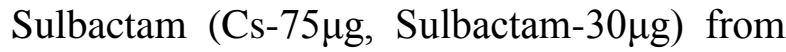
Pfizer were also included to study the antibiotic susceptibility pattern of these isolates.

Commercially available antibiotic disc were checked for quality using standard strains and then used for the test. For doing antibiogram, 4 -5 well demarcated colonies from the culture were inoculated into nutrient broth and incubated at $37^{\circ} \mathrm{C}$ till the density of the suspension to be inoculated matched the opacity standard of $0.5 \mathrm{McFarland}$ (barium sulphate suspension) turbidity. A lawn culture of the test organism was made on MHA plate with a sterile cotton swab soaked in the broth, after removing the excess broth by pressing against the sides of the test tube. The plates were incubated at $37^{\circ} \mathrm{C}$ for 18 to 24 hours after which the zone of inhibition was measured with calipers and compared with control strains as per Clinical and Laboratory Standards Institute (CLSI) guidelines. The reading of the test strain was taken only if the control strains showed satisfactory zone size in accordance with the CLSI (NCCLS) guidelines.

\section{Double Disc Synergy Test (DDST)}

In DDST, either enhancement of the zone size (for the III generation cephalosporins) of the antibiotic in the presence of clavulanate or clear extension of the edge of the inhibition zone of any of the antibiotic towards the disc containing clavulanic acid was interpreted as an indication of ESBL production.

The test organism was grown overnight at $37^{\circ} \mathrm{C}$ on nutrient agar plate. Isolated colonies of organism was inoculated into peptone water and incubated at $37^{\circ} \mathrm{C}$ and the turbidity was adjusted to 0.5 Macfarland standards. A lawn culture of the test organism was made on MHA plate with a sterile cotton swab soaked in the broth, after removing the excess broth by pressing against the sides of the test tube.

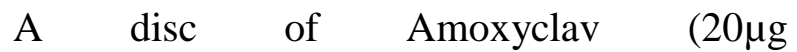
Amoxycillin/10 $\mu$ g Clavulanic acid) was placed in the center of the lawn culture, on the three sides of this disc at a distance of $30 \mathrm{~mm}$ from the edge of the above disc; discs containing Ceftazidime, Cefotaxime and Ceftriaxone were placed. Plates were then incubated at $37^{\circ} \mathrm{C}$ for 18 to 24 hours.

The isolates interpreted as ESBL if the inhibition zone around one or more cephalosporin disc was extended on the side nearest to the Amoxyclav disc or clear extension of the edge of the inhibition zone of any of the antibiotic disc towards the Amoxyclav disc. If there is no extension of the zone, the test was repeated by reducing the distance between the discs to $20 \mathrm{~mm}$. The test was considered negative if there was no distortion or synergy. 
Inhibitor potentiated disc diffusion technique

The test organism was grown at $37^{\circ} \mathrm{C}$ on a nutrient agar plate incubated overnight. Isolated colonies of the organism were inoculated into peptone water and incubated at $37^{\circ} \mathrm{C}$ and the turbidity adjusted to 0.5 Macfarland standards.

A lawn culture of the test organism was made on the MHA plates with a sterile cotton swab soaked in the broth, after removing the excess broth by pressing against the sides of the test tube and the following discs were placed,

Ceftazidime $(30 \mu \mathrm{g})$ / Ceftazidime-clavulanate $(30 \mu \mathrm{g}+10 \mu \mathrm{g})($ Himedia)

Cefaperazone $(30 \mu \mathrm{g})$ / Cefaperazonesulbactam $(30 \mu \mathrm{g}+75 \mu \mathrm{g})$ (Himedia)

Piperacillin $(10 \mu \mathrm{g}) /$ Piperacillin-tazobactam $(10 \mu \mathrm{g}+100 \mu \mathrm{g})(\mathrm{BBL}, \mathrm{USA})$

After placing these discs, the plates were incubated at $37^{\circ} \mathrm{C}$ for 18 to 24 hours.

Zone diameter of the antibiotic (alone) and antibiotic with the inhibitor combination were compared. If the difference in zone size was $\geq$ $5 \mathrm{~mm}$ it was indicative of ESBL production.

\section{Results and Discussion}

The present study was carried out from November 2004 to April 2005 in the Department of Microbiology, Sri Ramachandra Medical College and Research Institute which is a tertiary care centre. A total of50 non repetitive isolates of Klebsiella obtained from patients admitted in the ICU for more than 48 hours were included in the study. The samples for study were collected from patients with underlying cardiac or renal diseases, malignancy, diabetes with complications, road traffic accidents etc. They subsequently acquired infection with Klebsiella at varying periods after a minimum of 48 hours of hospitalization.
There was almost an equal distribution of the isolates among the genders, the males constituting $29(58 \%)$ and females $21(42 \%)$ of the total number (Figure 1). The male: female ratio was 1.04: 1 .

The demographic profile of the study subjects is shown in figure 2. The age distribution shows that infection with Klebsiella was common in middle and older age group. Among the total $(\mathrm{n}=50)$, majority of the patients $(54 \%)$ were between 31 to 60 years of age.

The distribution of isolates in various samples is shown in figure 3. Majority of the isolates were obtained from respiratory specimens, blood followed by urine and exudates samples. The rate of isolation of Respiratory isolates accounted for $40 \%(n=34)$ which include endotracheal secretions, bronchial wash, endotracheal tube tips and sputum and the isolation rate from blood accounted for 36 $\%(\mathrm{n}=18)$ from urine samples was $14 \%(\mathrm{n}=7)$ followed by exudates which constituted $10 \%$ ( $\mathrm{n}=5$ ) which included pus, wound swab, drain tips.

The respiratory isolates were recovered from bronchial wash and endotracheal tube secretion from patients who were on ventilatory support. The outcome of these patients was fatal which is attributed to the underlying illness. The risk factors in these patients were stay in ICU, intubation and exposure to multiple antibiotics.

The organism isolated from urine was mostly isolated from male patients with advanced age, diabetes mellitus and underlying renal disease.

The isolates obtained from exudates samples were from elderly diabetic male patients with severe underlying illness such as epidural hemorrhage, chronic kidney disease and road traffic accident. There were surgical 
interventions in all these patients. Risk factors in these patients were presence of indwelling devices, recent surgery and use of multiple antibiotics.

The isolates were identified based on Bergey's manual of determinative bacteriology Annexure III. Gram stain showed gram-negative bacilli which were capsulated, uniformly stained with parallel sides and rounded ends. They produced the characteristic large grey mucoid colonies on blood agar and lactose fermenting large pink mucoid colonies on MacConkey agar. The isolates had gas production with acid slant and acid butt in TSI medium, fermented glucose both aerobically and anaerobically and were nonmotile with mannitol fermented.

The various isolates of the genus Klebsiella obtained in the present study are depicted in figure 4. Out of the total Klebsiella isolates, Klebsiella pneumoniae subspecies pneumoniae was the commonest isolate $34(68 \%)$ followed by Klebsiella oxytoca 9(17\%), Klebsiella planticola in 4(9\%) and Klebsiella pneumoniae subspecies ozaenae in $3(6 \%)$. The species wise distribution of the isolate from various clinical samples and their correlation is given in table 1 and figure 5

In the present study, all Klebsiella pneumoniae subspecies pneumoniae were found to produce gas and ferment all the carbohydrates tested. None of the isolates were able to produce indole and acid during fermentation of glucose in MR tests. All the isolates were able to produce acetoin, utilize citrate, produce alkali, and reduce nitrate and decarboxylate the amino acid lysine.

All the Klebsiella oxytoca in the study were able to produce indole, ferment all the carbohydrates with gas production, reduce nitrates, and produce alkali and acetoin. Most of them decarboxylated the amino acid lysine and did not produce acid from glucose in MR test.

Most strains of Klebsiella planticola did not produce indole and none of the isolates were able to ferment dulcitol. All the isolates were found to ferment glucose in MR tests, produce acetoin, utilize citrate, reduce nitrate, and form alkali and decarboxylate lysine.

None of the isolates of Klebsiella pneumoniae subspecies ozaenae was able to produce indole, acetoin in VP test and ferment the carbohydrates sucrose and dulcitol. All the isolates were able to reduce nitrates, decarboxylate lysine and utilize citrate. However, none of the strains produced alkali by the urease test.

Total number of isolates from MICU was $80 \%(\mathrm{n}=40)$ and SICU wards $20 \%(\mathrm{n}=10)$ $(\mathrm{p}<0.05)$ (Figure 6). In the ICU, majority of the isolates were from respiratory samples followed by blood, urine and exudate specimens. The immune status of each patient was assessed depending on the following conditions like diabetes mellitus, malignancy, intake of steroids, HIV and HBsAg. The breakup of the immunocompromised states is shown in figure and 7.

One of the above immunocompromised factor was found in $39 \%(n=19)$. About $12 \%(n=6)$ of patients had more than one immunocompromised factor. Of the total, 49 $\% \quad(n=24) \quad(\mathrm{p}<0.05) \quad$ patients were immunocompetent. Of the $39 \%(\mathrm{n}=19)$ immunocompromised patients, $43 \% \quad(\mathrm{n}=8)$ had diabetes, malignancy was recorded in $2 \%$ $(\mathrm{n}=1)$, long term steroid use / immunosuppressant drugs was seen in $13 \%$ $(n=3)(p<0.01)$ of the patients, and 6\% $(n=1)$ patients were on dialysis.

The predisposing risk factors $(\mathrm{p}<0.01)$ like any surgical procedures, vascular line access, 
ventilators, urinary catheters, presence of any drain tubes etc were analyzed and given in table 2 .

About $57 \%$ patients $(n=29)$ had more than one intervention ( $\mathrm{p}<0.001)$. Only 15\% ( $\mathrm{n}=19)$ had no intervention. History of previous or recent surgical procedures was recorded in $53 \%$ of patients. Prior antibiotic therapy was considered to be the most important risk factor for the acquisition of Klebsiella infection among hospitalized patients. Majority of the patients $31 \%$ had at least one antibiotic followed by $30 \%$ patients with three antibiotics. Only one patient $(0.7 \%)$ had no antibiotic therapy. However, in $37 \%$ patients there was usage of 3 to 5 antibiotics. This might have been due to the frequent changeover of different antibiotic classes.

In the present study, a total of 18 blood samples of which $K$. pneumoniae was 14 and $K$. oxytoca collected were 7 . Figure 8 shows the distribution of isolates in blood samples.

In the present study, a total of 20 respiratory tract specimens which includes E.T. tip $(n=11)$, E.T.secretion $(n=4)$, sputum $(n=1)$ and bronchial wash $(\mathrm{n}=4)$ were collected. $K$. pneumoniae was found to be isolated in all the respiratory samples (Table 3 ).

\section{Antibiotic susceptibility pattern}

The susceptibility exhibited by each isolate is shown in table 4 . The various classes of antibiotics tested are as follows, betalactam antibiotics such as Ampicillin, Piperacillin, Cephalosporins (Cefazolin, Cefuroxime, Ceftazidime, Cefotaxime, Ceftriaxone, and Cefaperazone), (Ciprofloxacin), (Amikacin), fluoroquinolones Aminoglycosides inhibitor combinations (PiperacillinTazobactam, Cefaperazone-Sulbactam) and Carbapenem (Imipenem). In the present study, all the isolates $(n=50)(100 \%)$ were found resistant to the betalactam antibiotics such as Ampicillin and Piperacillin. Of the total 50 strains, Amikacin resistance was found in $47 \%$ isolates of which $48 \%$ were found in Klebsiella pneumoniae, followed by 52\% (Klebsiella oxytoca. Amikacin resistance was also found in Klebsiella ozaenae and Klebsiella planticola which accounted for $38 \%$ and $42 \%$ respectively. However, Amikacin sensitivity was observed in $53 \%$ Klebsiella isolates of which $52 \%$ were in Klebsiella pneumoniae, $63 \%$ in Klebsiella ozaenae and $58 \%$ from Klebsiella planticola. There were only $48 \%$ isolates of Klebsiella oxytoca found to be sensitive.

Resistance to fluoroquinolones (Ciprofloxacin) was seen in $52 \%$ of the isolates and this includes 53\% Klebsiella pneumoniae, 48\% Klebsiella oxytoca, 67\% Klebsiella planticola and $25 \%$ isolates of Klebsiella ozaenae. Of the 50 strains, $48 \%$ isolates were sensitive to Ciprofloxacin. Maximum sensitivity was observed in Klebsiella pneumoniae $47 \%$ and least recorded in Klebsiella planticola 33\%.

In the present study, $30 \%$ isolates were recorded resistant to all betalactam antibiotics which include Ampicillin, Piperacillin, and all cephalosporins. Among the isolates, 54\% $(p<0.01)$ strains were found resistant to all the third generation cephalosporins. Maximum resistance to all the $3^{\text {rd }}$ generation cephalosporins was observed in Klebsiella planticola.

Among the $3^{\text {rd }}$ generation cephalosporins, ceftazidime was found to be the most resistant antibiotic $(\mathrm{p}<0.03)$ varying from $67 \%(\mathrm{n}=8)$ in case of Klebsiella planticola to $50 \%(\mathrm{n}=4)$ in Klebsiella ozaenae. Ceftazidime resistance in Klebsiella pneumoniae and Klebsiella oxytoca were $56 \%$ and $57 \%$ respectively. A high degree of resistance to Cefotaxime $(\mathrm{p}<0.01)$ was observed in Klebsiella oxytoca (62\%) next only to Klebsiella planticola (67\%). 
Resistance to Ceftriaxone and Cefaperazone $(\mathrm{p}<0.05)$ was almost equal in the various species of Klebsiella which includes $57 \%$ in both Klebsiella pneumoniae and Klebsiella oxytoca followed by $50 \%$ in Klebsiella ozaenae.

The susceptibility pattern of the betalactambetalactamase inhibitor combinations in the study was found to be variable. Of the total 50 strains, $66 \%$ and $62 \%$ of the isolates were found to be sensitive to PiperacillinTazobactam $(\mathrm{p}=0.01)$ and CefaperazoneSulbactam $\quad(\mathrm{p}<0.05) \quad$ combinations. For Klebsiella pneumoniae and Klebsiella oxytoca, the susceptibility to PiperacillinTazobactam was $64 \%$ and $71 \%$ respectively. But slightly lowered susceptibility rates were recorded with Cefaperazone- Sulbactam for both Klebsiella pneumoniae (60\%) and Klebsiella oxytoca (67\%). All the isolates were found to be sensitive to Carbapenems (100\%).

Of the 50 nonrepetitive isolates from the hospitalized inpatients, strains which showed a zone diameter of $\leq 22 \mathrm{~mm}$ for ceftazidime and / or $\leq 27 \mathrm{~mm}$ for cefotaxime or found resistant to any one of the third generation cephalosporin on routine antibiotic susceptibility testing by Kirby-Bauer disc diffusion technique were subjected for identification of ESBL.

Of the 50 resistant isolates Klebsiella pneumoniae were $68 \% \quad(\mathrm{n}=34)$ and other Klebsiella species were $32 \% \quad(\mathrm{n}=16)$. The resistance pattern of the 50 isolates to different antibiotics by Kirby-Bauer disc diffusion techniques is shown in figure 9. All the resistant isolates exhibited different patterns of cross resistance to different classes of antibiotics.

Screening for ESBL based on Ceftazidime was performed by agar dilution technique. All the 50 strains on screening with double disc synergy test $21 \% \quad(n=21)$ of the isolates showed either enhancement of the zone size in the presence of Clavulanic acid or clear extension of the edge of the inhibition zone of any of the third generation cephalosporin towards the Clavulanic acid disc (Figure 10).

Inhibitor potentiated disc diffusion test was done using three drug/inhibitor combination. An increase in zone size by $\geq 5 \mathrm{~mm}$ with Ceftazidime/Clavulanic acid, Piperacillin/Piperacillin-Tazobactam and Cefaperazone/Cefaperazone-Sulbactam was observed in $84 \%(n=42), 100 \%(n=50)$ and $94 \% \quad(n=47) \quad(p<0.05)$ of the isolates respectively (Table 5).

Klebsiella pneumoniae is the species most frequently isolated in clinical laboratories. In the present study also, K. pneumoniae was the most common species isolated from clinical samples. Among the total 50 isolates, the isolation rate of Klebsiella species from the intensive care units includes Klebsiella pneumoniae was $68 \%$ followed by $K$. oxytoca $17 \%$, K. planticola $9 \%$ and finally $K$. ozaenae 6\%. In a study done by Arora et al., 2003 and Subha et al., 2003, K. pneumoniae was isolated at the rate of $84 \%$ and $83 \%$ from various clinical samples followed by $K$. oxytoca in $16 \%$ and $17 \%$ in their study. David Livermore and Yuan, 1996 reported 74\% K. pneumoniae and $26 \% \mathrm{~K}$. oxytoca followed by only 2 isolates $(0.2 \%)$ of $K$. ozaenae. However Priya Datta et al., 2004 isolated only $35.7 \%$ of $K$. pneumoniae followed by $4 \% \mathrm{~K}$. oxytoca from clinical samples.

David Livermore and Gioia Babini, 2000 reported $70.6 \% \mathrm{~K}$. pneumoniae, $26.6 \% \mathrm{~K}$. oxytoca and only $(2.8 \%)$ one isolate of $K$. ozaenae recovered from patients admitted in ICUs of 21 hospitals.

In our study $K$. pneumoniae was isolated at the rate of $68 \%$ from various clinical samples that included urine, exudates and blood, and 
respiratory tract specimens. Among the Klebsiella pneumoniae isolates $41 \%$ (35) were from SICU and 59\% (51) were from MICUs.

Subha et al., (2003) reported $83 \% \quad K$. pneumoniae at the rate of $21 \%$ in blood, $58 \%$ in urine and $4 \%$ in respiratory tract specimens. In a study done by the same author in 2001 the rate of isolation of $K$. pneumoniae $(84 \%)$ from various clinical samples was found to be $22 \%$ (17) blood, $57 \%$ (43) urine, $18.4 \%$ (14) from stool, and $2 \%$ (2) from throat swab.

Supriya Tankhiwale et al., 2005 and Robert Lewis et al., 1978 who reported $37 \%$ and $38 \%$ of $K$. pneumoniae from urine samples. However, Subha et al., 2001 had a slight increase $(56 \%)$ in the isolation rate of $K$. pneumoniae from urine.

Out of the $44 \%$ Klebsiella species isolated in our study, $16 \%$ were from SICU and $84 \%$ were from MICU wards. The presence of indwelling catheter was observed in $48 \%$ of the patients in both ICU setting. Age, presence of urinary catheters, BPH, CRF, surgical procedures, urogenital abnormality, prolonged hospital stay and exposure to antibiotics were found to be the associated risk factors for isolation from urinary tract.

Among the $16 \%$ of Klebsiella pneumoniae isolated from respiratory tract samples, a high rate of isolation from endotracheal tube tips was noted $82 \%$. The rate of isolation from endotracheal secretion, bronchial wash and sputum were $14 \%, 14 \%$ and $7 \%$ respectively. In our study, $15 \%$ of the Klebsiella isolates were obtained from ventilated patients. HansJurgen Woske et al., (2001) recovered Klebsiella species from $9 \%$ of ventilated patients similar to the present study.

Of the $16 \%(\mathrm{n}=14)$ Klebsiella pneumoniae respiratory isolates, $93 \%(n=13)$ of them were obtained from patients on ventilatory support. The outcome in some of these patients was fatal which is attributed to the underlying illness. The risk factors observed in these patients were prolonged stay in ICU, intubation, exposure to multiple antibiotics and immunocompromised states like diabetes, steroid therapy.

In our study, $17 \%$ of $K$. pneumoniae was recovered from blood samples which were correlating well with the study done in North India by Manjula Mehta et al., 2005 who reported $15 \%$ of $K$. pneumoniae from blood samples. However, there was a slight variation in the isolation rate of Subha et al., 2001 who reported $22 \%$ of $K$. pneumoniae from blood. Thukral et al., (2005) isolated $K$. pneumoniae at the rate of $31 \%$ from wound swabs. In our study $41 \%$ of $K$. pneumoniae was isolated from various patients admitted in the ICU wards whereas only one isolate of $K$. pneumoniae was recovered from ICU in the study done by Thukral et al., 2005.

In our study $K$. pneumoniae was isolated from $18.6 \%$ of surgical wound infections however there was a slight increase in the incidence $(26.8 \%)$ of $K$. pneumoniae reported by Karyakarte et al., (1999) from surgical wounds.

K. oxytoca accounts for $17 \%$ of the clinical samples included in the present study. Subha et al., 2001 and Priya Dutta et al., (2004) isolated the organism from $15 \%$ and $4 \%$ of the total samples respectively.

Among the total, Klebsiella oxytoca was isolated from one blood sample from an ICU patient with demyelinating syndrome. The mortality rate associated with $K$. oxytoca in this study was $10 \%$ which is similar $(9 \%)$ to the mortality rate reported by Rong-Dih Lin et al., (1997). In our study the risk factors associated with $K$. oxytoca infection includes catheterization (52\%) intubation (33\%), 
steroid medication $(10 \%)$, diabetic $(52 \%)$ and $67 \%$ patients who had undergone surgery recently. The use of more than 2 antibiotics was recorded in $43 \%$ of the patients and presence of i.v. line found in $76.1 \%$ of the patients.

K.ozaenae was isolated from urine3 (100\%) specimens. Katherine Murray et al., (1981) isolated $40 \%$ of $K$. ozaenae from various clinical samples which included exudates, urine and blood. $K$. ozaenae associated bacteremia has been reported only in one case in our study.

In this study, $K$. planticola was isolated from only $9 \%$ of the total clinical specimens which include $100 \%$ (4) urine sample. Podschun et al., (1998) and Brian Mee et al., (1997) have isolated only $8.7 \%$ and $9 \%$ of $K$. planticola from clinical specimens. Freney et al., (1986) has reported $18 \%$ to be $K$. planticola isolated from 14 tracheal aspirates, 3 urine, 2 sputum, 2 throat swabs, 1 CSF, 1 nasal swab, and 1 venous catheter.

In the present study, $57 \%$ of $K$. pneumoniae was found to be resistant to any one of the $3^{\text {rd }}$ generation cephalosporins. However, Hansotia et al., (1997) isolated $26 \%$ of $K$. pneumoniae that were found resistant to $3^{\text {rd }}$ generation cephalosporins. The prevalence of ESBL producing Klebsiella species was found to be $58 \%$ in our study. Mathur et al., (2002) reported a higher proportion of ESBL positive Klebsiella species (80\%). Sumeeta Khurana et al., (2002) reported $38.5 \%$ of Klebsiella species to produce ESBL. Das et al., (2004) reported ESBL in Klebsiella species to be $76.34 \%$

In a study in south India done by Subha et al., 2002 ESBL mediated resistance was $25.8 \%$ and in another study by Hansotia et al., (1997) a very low prevalence of $6 \%$ was reported.
Emily Hyle et al., (2005) observed ESBL in $K$. pneumoniae to be $50.7 \%$ and $5.8 \%$ in $K$. oxytoca. Priya Dutta et al., (2004) reported $35.7 \% \mathrm{~K}$. pneumoniae and $16.6 \%$ of $K$. oxytoca as producing ESBL. In the present study $K$. pneumoniae harbouring ESBL were $66 \%$ and $K$. oxytoca with ESBL were $17.5 \%$.

In our study ESBL producing $K$. pneumoniae was found in $66 \%$ of the isolates. Huseyin Tash et al., (2005) reported the prevalence of ESBL producers among $K$. pneumoniae to be $57.1 \%$.

In the ICUs, ESBL producing $K$. pneumoniae isolates were more frequently detected in blood $(46 \%)$ and respiratory tract samples (37\%) similar to the study done by Carmen Pena et al., (1998). In MICUs, isolates were detected in urine (56\%) and exudate samples (40\%) similar to the study of Carmen Pena et al., 1998. In the present study ESBL producing $K$. pneumoniae was isolated from blood $(\mathrm{n}=14)$ respiratory tract specimen $(\mathrm{n}=20)$. In a study from North India, performed by Shukla et al., (2004) similar rate of isolation was recorded in blood isolates $(24 \%)$ in contrast to variable values obtained for urine $(20.5 \%)$ and pus $(36.1 \%)$. This variation might be due to the selection of the study group involved.

Carmen Pena et al., 1998 reported 35\% ESBL producing $K$. pneumoniae isolates. The author also found the isolates to be more frequently recovered from blood (40\%) and respiratory samples (26\%) in an ICU setting, whereas the non-ICU wards showed greater incidence of ESBL producing $K$. pneumoniae in urine samples $(55 \%)$ followed by surgical wound samples (34\%).

In the present study, ESBL positive $K$. pneumoniae found to be $68 \%$. Presence of renal failure, obstructive uropathy which included stricture urethra and benign prostatic hypertrophy were found to be the risk factors 
responsible for the acquisition of ESBL producing $K$. pneumoniae infection. $K$. pneumoniae strains are mostly isolated from urine but unexpectedly, the percentage of $K$. pneumoniae strains resistant to extended spectrum cephalosporins and Aztreonam was lower for UTI than for other infections, especially bacteremia and respiratory tract infections. The ESBL positivity in urinary isolates of $K$. pneumoniae in Latin American hospitals, USA and Canada was reported as $37.7 \%, 6.4 \%$, and $6.2 \%$ respectively.

In our study, the isolation rate of ESBL producing $K$. pneumoniae from blood was 24\% whereas David Paterson et al., 2004 isolated $30.8 \% \mathrm{~K}$. pneumoniae with ESBL. In a report by Lautenbach et al., (2001), only $9.1 \%$ of the patients had blood stream infections with ESBL producing $K$. pneumoniae.

In our study, the overall mortality rate due to K. pneumoniae was $8.1 \%(\mathrm{n}=7)$, of which 5 isolates of $K$. pneumoniae were found to have ESBL. In a report of 216 patients with $K$. pneumoniae bacteremia, Paterson et al., (2004) indicated that mortality rate was $46 \%$ for 32 patients with bacteremia caused by ESBL producing $K$. pneumoniae strains and $34 \%$ for patients with bacteremia caused by ESBL nonproducing $K$. pneumoniae strains.

Reduction in the use of betalactam antibiotics containing an oxyiminogroup and infection control measures may reduce the spread of ESBL producing organisms within a hospital (David Paterson et al., 2004). Among the ESBL isolates of our study, the resistance among aminoglycosides and quinolones was found to be $73 \%$ and $78 \%$. Resistance to $3^{\text {rd }}$ generation cephalosporins was found in $97 \%$ isolates for ceftazidime, $95 \%$ strains to cefotaxime and $99 \%$ isolates for ceftriaxone and cefaperazone. There were $62 \%$ and $32 \%$ of the isolates found sensitive to cefoxitin and cefepime.
As observed by Hernandez et al., (2005), the present study also showed the concurrence of ciprofloxacin resistance with ESBL production, particularly in isolates of $K$. pneumoniae. Cross resistance to other antibiotic classes was common, in our study $73 \%$ and $78 \%$ of strains were resistant to amikacin and ciprofloxacin compared to $41 \%$ and $100 \%$ resistance observed by Janis Weiner et al., (1999) for aminoglycosides and quinolones.

Subha et al., (2002) from Chennai had reported $75 \%$ of the isolates to be resistant to amikacin which is comparable to the present study where there were $73 \%$ of the isolates of $K$. pneumoniae harbouring ESBLs found resistant to amikacin.

In concurrence with the present study, Jerestin Hansotia et al., 1997 observed variable sensitivity to the non-betalactam like Amikacin (64\%) and Ciprofloxacin (35\%).

Mathur et al., (2002) reported 63\% of the isolates resistant to amikacin and $81 \%$ to ciprofloxacin similar to the present study.

In our study Amikacin and Ciprofloxacin resistance was seen in $74 \%$ isolates compared to $61 \%$ and $31 \%$ reported by Livermore and Gioia Babini (2000).

In the present study ESBL mediated resistance against cephalosporins was found in $51 \%$ of $K$. pneumoniae isolates which is much less than the study done by Shukla et al., (2004) where $72 \%$ strains of $K$. pneumoniae were involved.

In the present study, all isolates were susceptible to Imipenem and resistant to ampicillin, piperacillin, astreonam, cefazolin and cefuroxime. Synergy to clavulanate was found in $43 \%$ of the isolates and $57 \%$ had no synergy. 
In our study only $43 \%$ of the isolates were detected as ESBLs by the DDST compared to $27.3 \%$ isolates detected by DDST by Shukla et al., (2004). In our study 42 strains were positive by DDST. 8 strains which were DDST negative could have had the AmpC profile, porin deficiency or other factors operating. Of these 42 strains, 27 were cefoxitin resistant, thus reflecting the possibility of chromosomal AmpC (MartinezMartinez et al., 1996). The sensitivity of DDST of the present study was only $43 \%$ similar to Priya Datta et al., (2004). Vercauterens et al., (1997) observed variable degrees of $79 \%$ and $93 \%$ sensitivity to the DDST.

Table.1 Correlation of clinical samples and species of Klebsiella

\begin{tabular}{|l|l|l|l|l|}
\hline samples & $\begin{array}{l}\text { Klebsiella } \\
\text { pneumoniae }\end{array}$ & $\begin{array}{l}\text { Klebsiella } \\
\text { oxytoca }\end{array}$ & $\begin{array}{l}\text { Klebsiella } \\
\text { ozaenae }\end{array}$ & $\begin{array}{l}\text { Klebsiella } \\
\text { planticola }\end{array}$ \\
\hline Respiratory & 20 & 0 & 0 & 0 \\
\hline Blood & 14 & 7 & 0 & 0 \\
\hline Urine & 0 & 1 & 3 & 4 \\
\hline Exudate & 0 & 1 & 0 & 0 \\
\hline
\end{tabular}

Table. 2 Predisposing risk factors

\begin{tabular}{|l|c|c|}
\hline Type of Interventions & $\begin{array}{c}\text { Numbers } \\
(\mathrm{n}=127)\end{array}$ & Percentage \\
\hline Line access & 42 & $84 \%$ \\
\hline Urinary catheter & 25 & $50 \%$ \\
\hline Ventilator & 18 & $35 \%$ \\
\hline Others (dialysis, wound drain) & 8 & $17 \%$ \\
\hline More than one interventions & 29 & $57 \%$ \\
\hline
\end{tabular}

Table.3 Distribution of isolates among respiratory samples

\begin{tabular}{|c|c|c|c|c|c|}
\hline Organism & E.T. tip & E.T.secretion & $\begin{array}{c}\text { Bronchial } \\
\text { wash }\end{array}$ & Sputum & Total \\
\hline K. pneumoniae & 9 & 6 & 4 & 1 & 14 \\
\hline
\end{tabular}


Table.4 Antibiotic Susceptibility Pattern of Klebsiella species

\begin{tabular}{|l|c|c|c|c|c|c|c|c|}
\hline \multirow{2}{*}{ Antibiotic } & $\begin{array}{r}\text { Klebsiella } \\
\text { pneumoniae }(\mathbf{n = 3 4 )}\end{array}$ & $\begin{array}{c}\text { Klebsiella } \\
\text { planticola } \\
\text { N=4 }\end{array}$ & $\begin{array}{c}\text { Klebsiella oxytoca } \\
(\text { n=9) }\end{array}$ & $\begin{array}{c}\text { Klebsiella ozaenae } \\
(\text { n=3) }\end{array}$ \\
\cline { 2 - 9 } & $\mathbf{S}$ & $\mathbf{R}$ & $\mathbf{S}$ & $\mathbf{R}$ & $\mathbf{S}$ & $\mathbf{R}$ & $\mathbf{S}$ & R \\
\hline Ampicillin & $0(0 \%)$ & $34(100 \%)$ & $0(0 \%)$ & $4(100 \%)$ & $0(0 \%)$ & $9(100 \%)$ & $0(0 \%)$ & $3(100 \%)$ \\
\hline Piperacillin & $0(0 \%)$ & $34(100 \%)$ & $0(0 \%)$ & $4(100 \%)$ & $0(0 \%)$ & $9(100 \%)$ & $0(0 \%)$ & $3(100 \%)$ \\
\hline Amikacin & $18(52 \%)$ & $16(48 \%)$ & $3(67 \%)$ & $1(33 \%)$ & $4(48 \%)$ & $5(52 \%)$ & $5(63 \%)$ & $3(38 \%)$ \\
\hline Ciprofloxacin & $16(47 \%)$ & $18(53 \%)$ & $1(33 \%)$ & $3(67 \%)$ & $5(52 \%)$ & $4(48 \%)$ & $6(75 \%)$ & $2(25 \%)$ \\
\hline Cefazolin & $6(17 \%)$ & $28(83 \%)$ & $1(33 \%)$ & $1(67 \%)$ & $1(19 \%)$ & $8(81 \%)$ & $0(0 \%)$ & $8(100 \%)$ \\
\hline Cefuroxime & $10(29 \%)$ & $24(71 \%)$ & $1(33 \%)$ & $1(67 \%)$ & $2(24 \%)$ & $7(76 \%)$ & $0(0 \%)$ & $3(100 \%)$ \\
\hline Ceftazidime & $19(56 \%)$ & $15(44 \%)$ & $1(33 \%)$ & $3(67 \%)$ & $3(43 \%)$ & $6(57 \%)$ & $1(50 \%)$ & $1(50 \%)$ \\
\hline Cefotaxime & $16(48 \%)$ & $18(52 \%)$ & $1(33 \%)$ & $3(67 \%)$ & $3(38 \%)$ & $6(62 \%)$ & $1(50 \%)$ & $1(50 \%)$ \\
\hline Ceftriaxone & $15(43 \%)$ & $19(57 \%)$ & $1(33 \%)$ & $3(67 \%)$ & $3(43 \%)$ & $657 \%)$ & $1(50 \%)$ & $1(50 \%)$ \\
\hline Cefaperazone & $15(43 \%)$ & $19(57 \%)$ & $1(33 \%)$ & $3(67 \%)$ & $3(43 \%)$ & $6(57 \%)$ & $1(50 \%)$ & $1(50 \%)$ \\
\hline $\begin{array}{l}\text { Cefaperazone- } \\
\text { Sulbactam }\end{array}$ & $20(60 \%)$ & $14(40 \%)$ & $3(67 \%)$ & $1(33 \%)$ & $6(67 \%)$ & $3(33 \%)$ & $3(100 \%)$ & $0(0 \%)$ \\
\hline $\begin{array}{l}\text { Piperacillin- } \\
\text { Tazobactam }\end{array}$ & $22(64 \%)$ & $12(36 \%)$ & $3(67 \%)$ & $1(33 \%)$ & $6(71 \%)$ & $3(29 \%)$ & $3(100 \%)$ & $0(0 \%)$ \\
\hline Imipenem & $34(100 \%)$ & $0(0 \%)$ & $4(100 \%)$ & $0(0 \%)$ & $9(100 \%)$ & $0(0 \%)$ & $3(100 \%)$ & $0(0 \%)$ \\
\hline aztreonam & $0(0 \%)$ & $34(100 \%)$ & $0(0 \%)$ & $4(100 \%)$ & $0(0 \%)$ & $9(100 \%)$ & $0(0 \%)$ & $3(100 \%)$ \\
\hline
\end{tabular}

Table.5 Inhibitor Potentiated Disc Diffusion Test

\begin{tabular}{|l|c|c|c|c|c|c|}
\hline \multirow{2}{*}{ Isolate } & \multicolumn{2}{c|}{$\mathrm{CaC}$} & \multicolumn{2}{c|}{ Tzp } & \multicolumn{2}{c|}{ Cfs } \\
\cline { 2 - 7 } & $>5 \mathrm{~mm}$ & $<5 \mathrm{~mm}$ & $>5 \mathrm{~mm}$ & $<5 \mathrm{~mm}$ & $>5 \mathrm{~mm}$ & $<5 \mathrm{~mm}$ \\
\hline K. pneumoniae & 28 & 6 & 34 & 0 & 31 & 3 \\
\hline Klebsiella oxytoca & 7 & 2 & 9 & 0 & 9 & 0 \\
\hline Klebsiella ozaenae & 3 & 0 & 3 & 0 & 3 & 0 \\
\hline Klebsiella planticola & 4 & 0 & 4 & 0 & 4 & 0 \\
\hline
\end{tabular}


Fig.1 Male-Female distribution

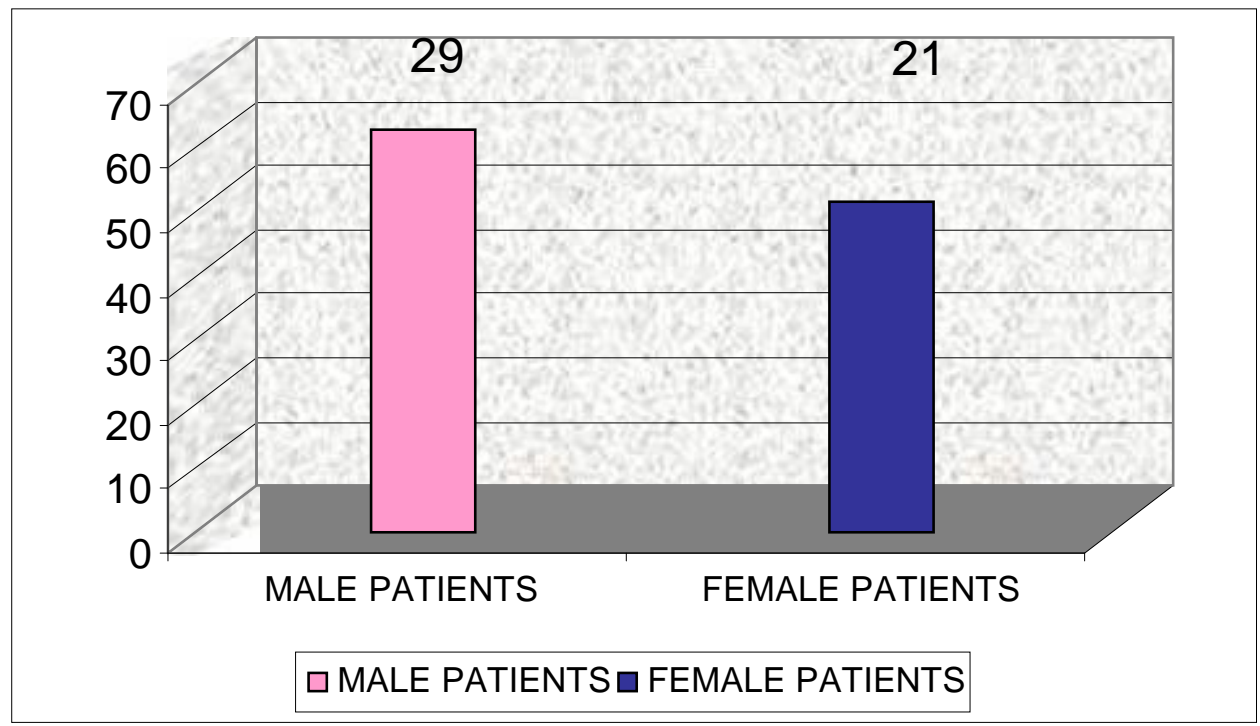

Fig.2 Demographic profile of study

\section{Demographic Profile of the study}

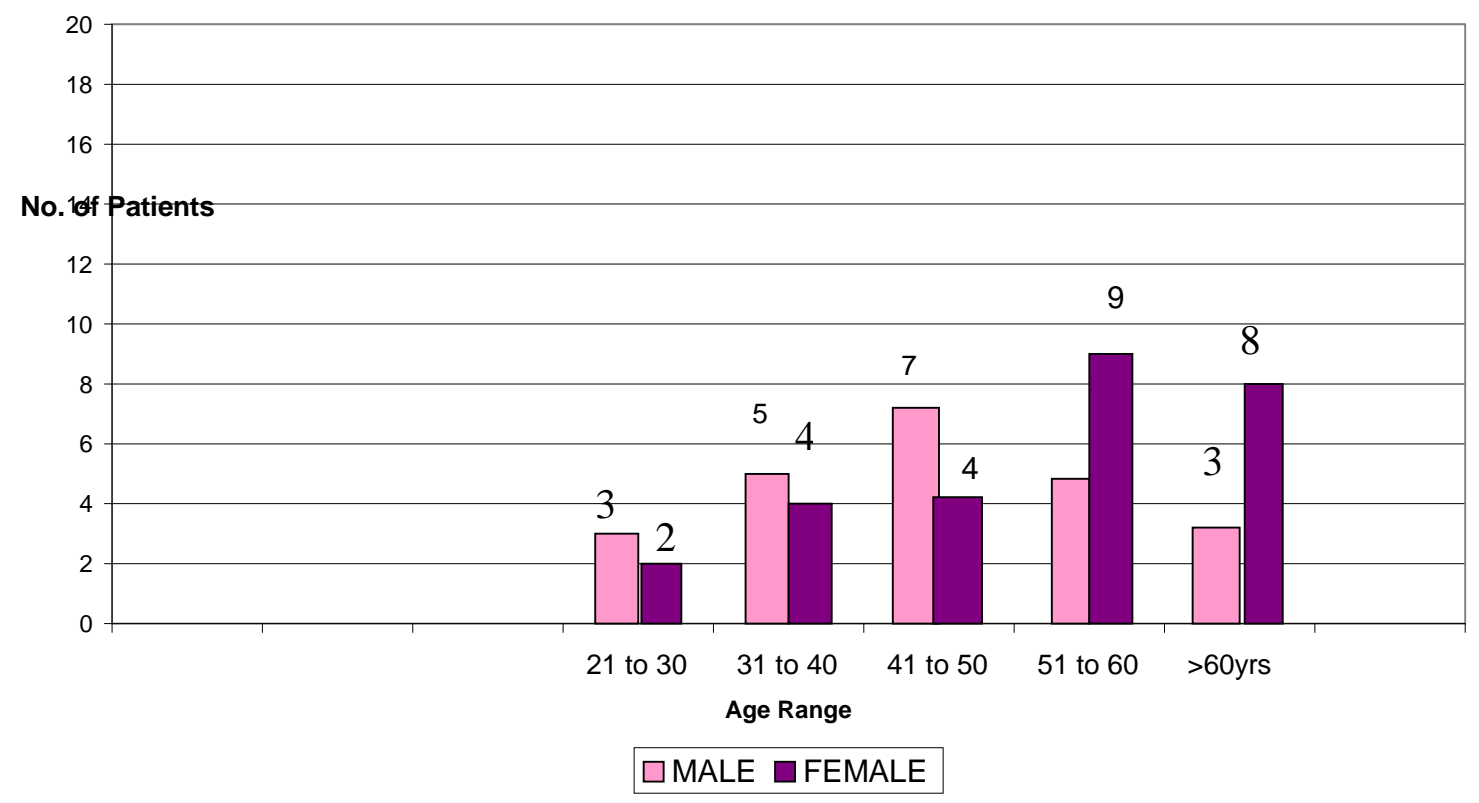


Fig.3 Distribution of isolates in various clinical samples

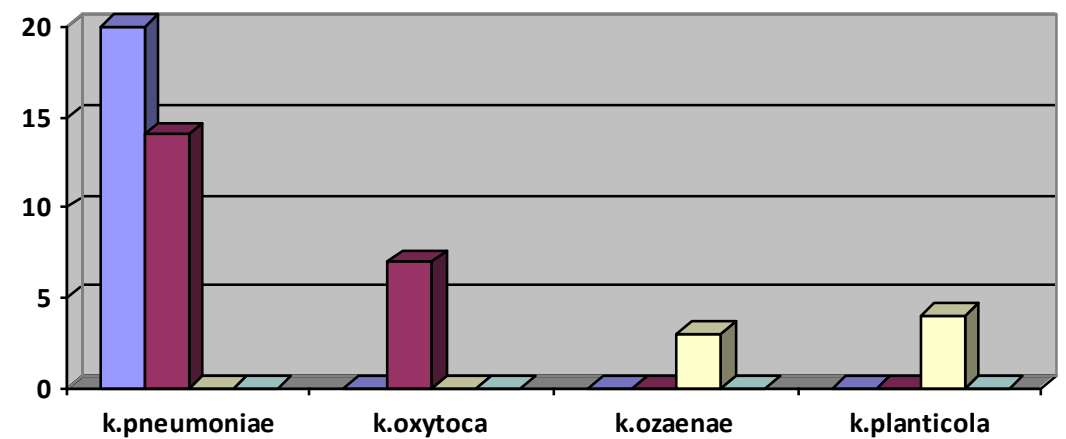

Fig.4 Klebsiella species isolated

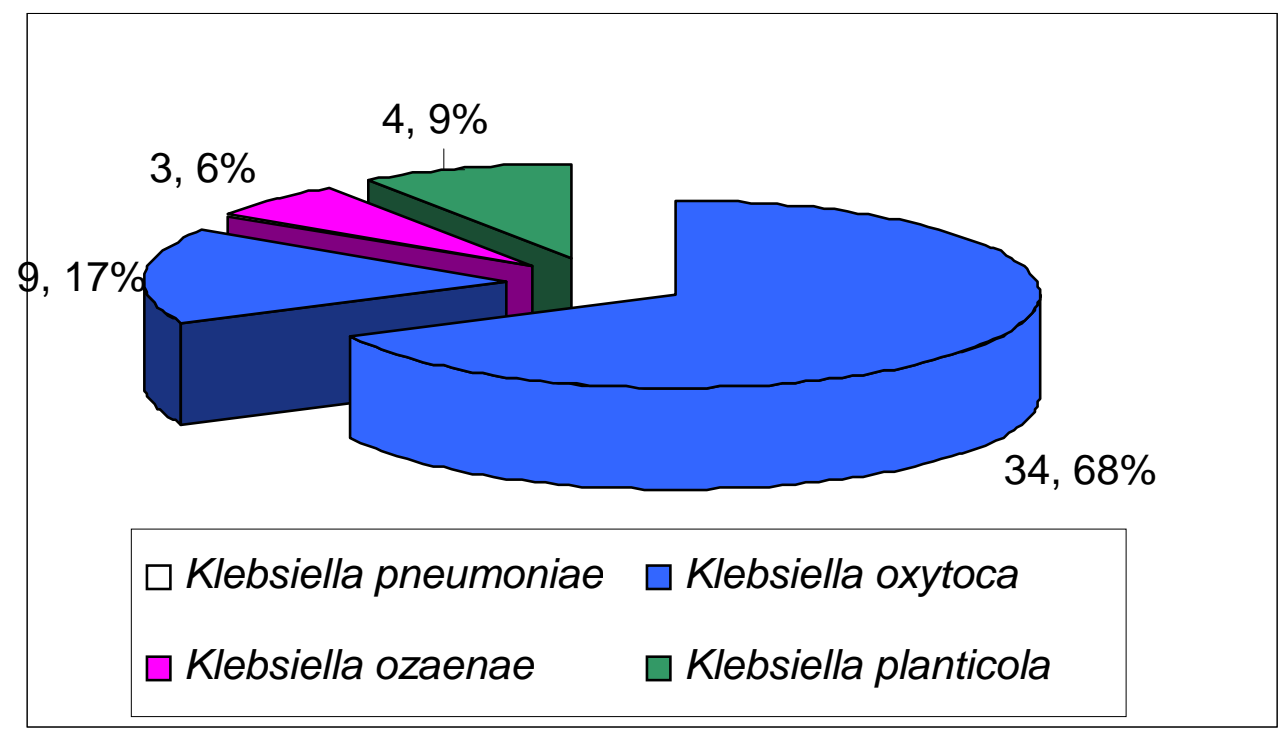

Fig.5 Correlation of clinical samples and species of Klebsiella

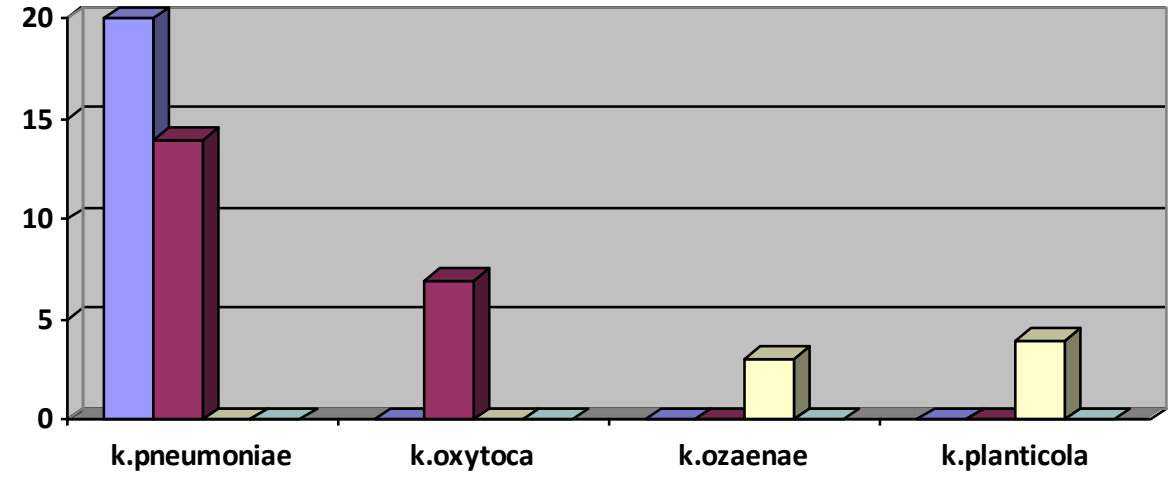


Fig.6 MICU /SICU distribution of Klebsiella

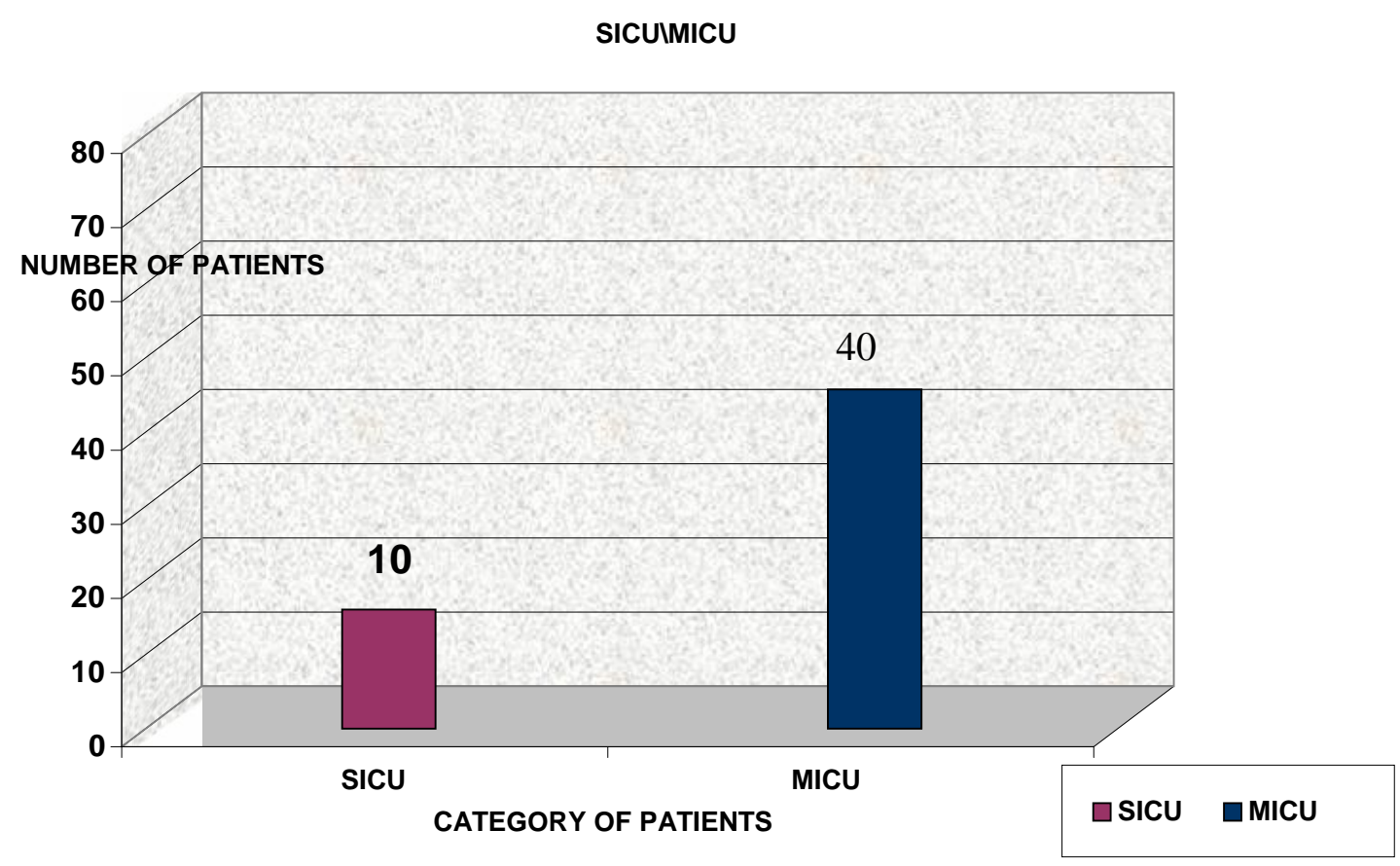

Fig.7 Immune status of the patient

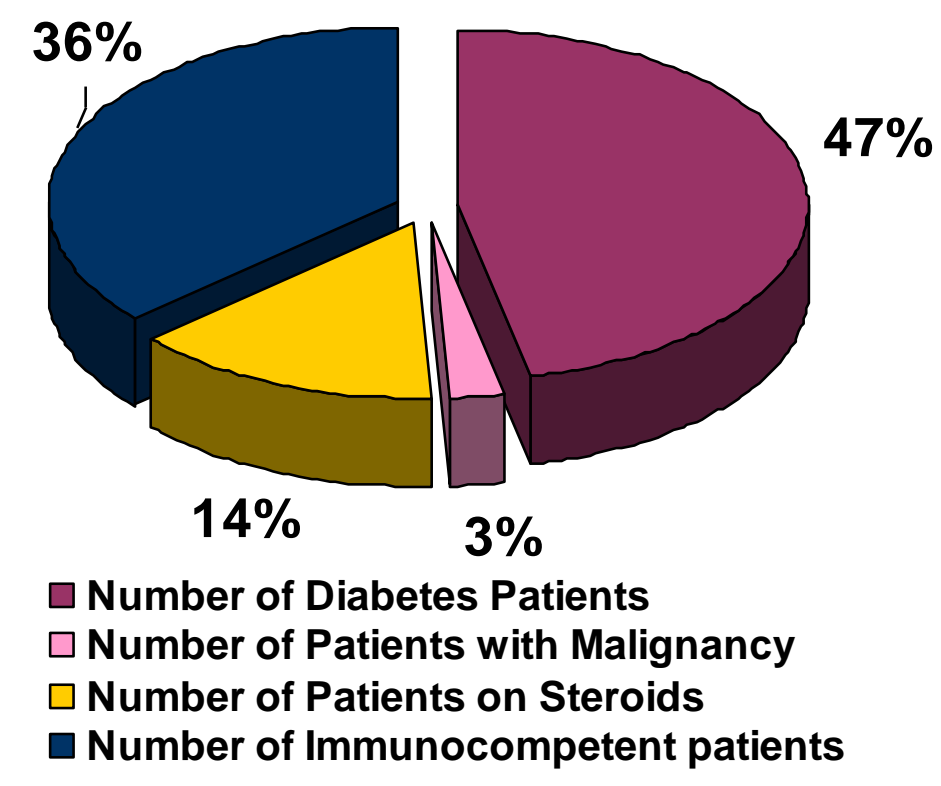


Fig.8 Distribution of isolates in blood samples

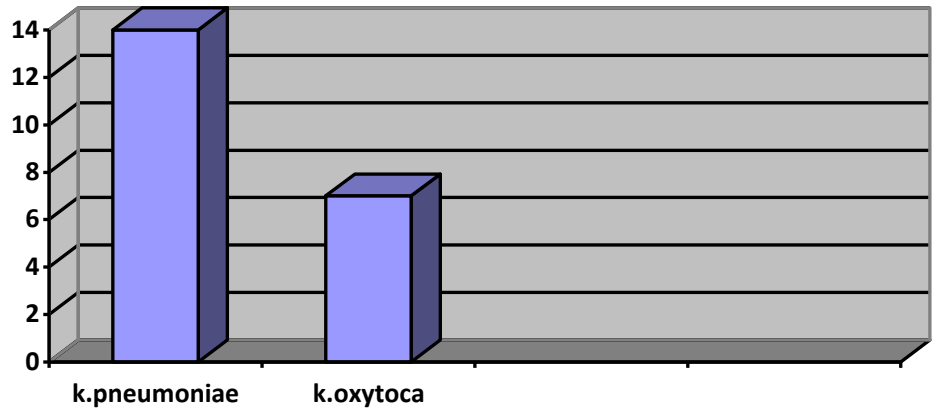

口numbers

Fig.9 Antibiotic Susceptibility pattern of ESBL isolates

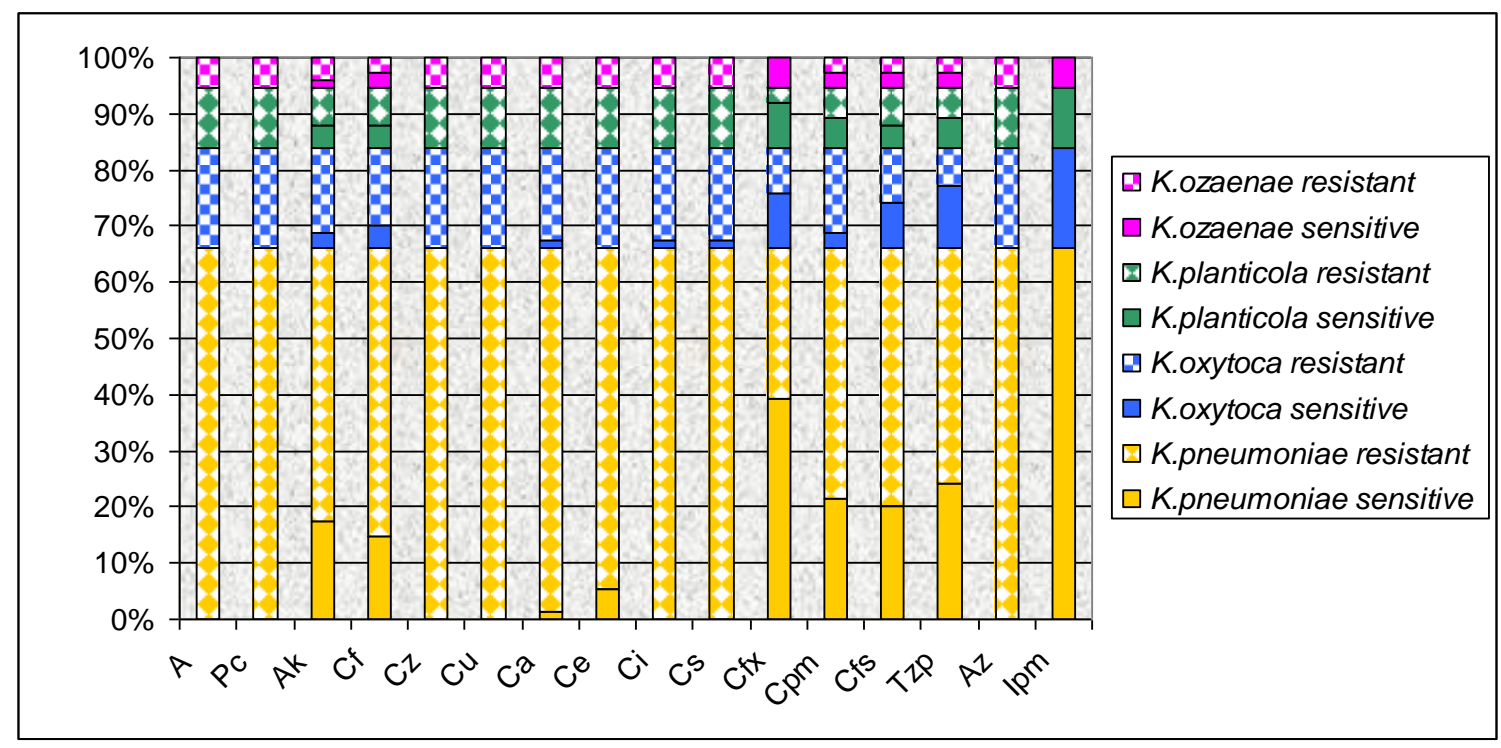

Fig.10 Klebsiella isolates positive for DDST

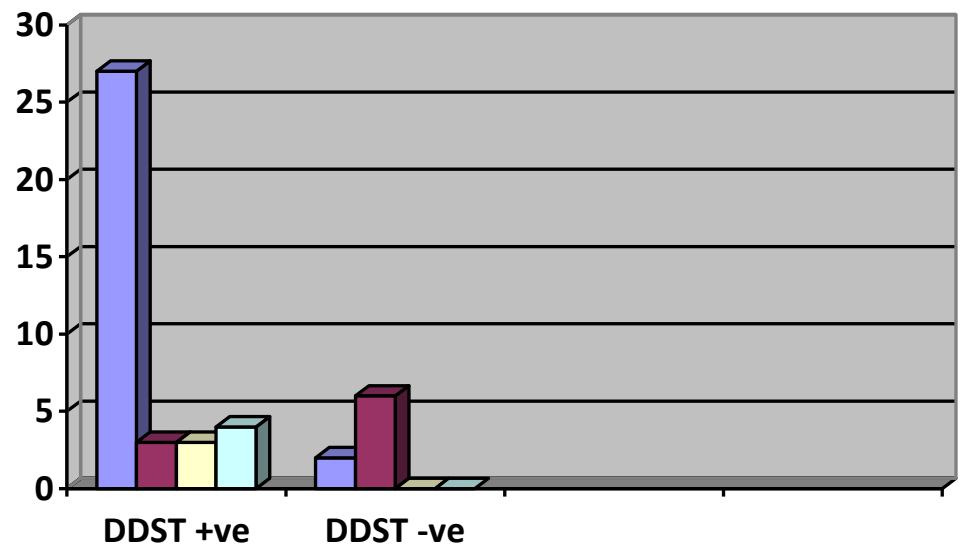

$\square$ K.pneumoniae
$\square$ K.oxytoca
$\square$ K.ozaenae
$\square$ K.planticola


The other confirmatory test used in this study was the IPDD test. CLSI states that this test can be performed with either ceftazidime/clavulanic acid or cefotaxime/clavulanic acid, but screening with both increases the sensitivity (NCCLS, 2002). In our study, $92 \%$ of the isolates were identified by IPDD test similar to $100 \%$ sensitivity observed by Ho et al., (1998).

In our study, 92\% strains of the total $74 \mathrm{ESBL}$ producers could be identified by IPDD test similar to Priya Datta et al., (2004). However, use of piperacillin/tazobactam was able to pick up more strains compared to ceftazidime/clavulanic acid and cefaperazone/sulbactam. Tazobactam is a more potent inhibitor of both plasmid and chromosomal mediated betalactamases.

In the present study, only $43 \%$ of Klebsiella species were susceptible to piperacillintazobactam combination compared to $35 \%$ and $70 \%$ susceptibility noted by Jill Rebuck et al., (2000) and Livermore et al., (1996). Likewise in our study also there were $57 \%$ of isolates found resistant to piperacillintazobactam and $65 \%$ to cefaperazonesulbactam.

Of the total ESBL producing Klebsiella isolates 68\% were resistant to Cefepime and all the isolates $100 \%$ resistant to Aztreonam. Nearly $68 \%$ of the ESBL producing $K$. pneumoniae were resistant to cefepime in our study but Cheol-In Kang et al., (2004) reported only $4.6 \%$ of the isolates to be resistant. Barroso et al., (2000) reported resistance to aztreonam in all the 138 isolates of his study.

In accordance with this study, Imipenem and cefepime were sensitive in $100 \%$ and $33 \%$ of the strains. Aksaray et al., (2000) found $98.6 \%$ and $70 \%$ of these strains to be sensitive to Imipenem and cefepime.
None of the Klebsiella isolates were found to be resistant to Imipenem which demonstrates the highest degree of sensitivity similar to Bradley Jett et al., (1995).

In conclusion,

Nosocomial Klebsiella infections continue to be a heavy burden on the economy and on the life expectancy of patients worldwide.

$\checkmark K$. pneumoniae is the most frequently isolated organism from clinical specimens and found to be associated with drug resistance.

Apart from K. pneumoniae and K. oxytoca, K.planticola in particular, has been isolated with increasing frequency from human infectious clinical samples.

In our study so far, ESBL producing Klebsiella strains have been susceptible to carbapenems.

\section{References}

Abdul Qavi, Janice Burns James, J., Rahal, Noriel Mariano, et al. 2005. Increased mortality associated with a clonal outbreak of ceftazidime resistant Klebsiella pneumonia: A case control study. Inf. Cont. and Hosp. Epi., 26: 63-68.

Aggarwal, A., Khanna, S., Arora, U. 2003. characterisation, biotyping,antibiogram nd klebocin typing of Klebsiella with special referrence to Klebsiella oxytoca., Indian J. Med. Sci., 57: 68-70.

Amita Jain, Indranil Roy, Mahendra, K., Gupta, Mala Kumar, et al. 2003. Prevalence of extended spectrum betalactamase producing gramnegative bacteria in septicemic neonates in a tertiary care hospital, J. Med. Microbiol., 52: 421-425.

Ana, C., Gales, Ronald, N., Jones, Kelly, A. Gordan, Helio, S., Sader et al. 2000. Activity and spectrum of 22 antimicrobial 
agents tested against UTI pathogens in hospitalised patients in Latin America: report from the second year of the SENTRY Antimicrobial Surveillance programme. J. Antimicrobial. Chemother., 45: 295-303.

Angel Asensio, Antonio Oliver,Javier Cobo, Fernando Baquero et al.,., Outbreak of multiresistant Klebsiella pneumoniae strain in intensive care unit: Antibiotic use as risk factor for colonisation and infection Clin. Infect. Dis., 30: 55-60.

Archana Gupta, Lisa Saiman, Janet Haas, David Rubenstein, et al. 2004. Outbreak of Extended spectrum betalactamase producing Klebsiella pneumoniae strain in aneonatal intensive care unit linked to artificial nails: Inf. Cont and Hosp.Epi., 25: 210-215.

Artemio Gonzalez-Vertiz, Jose, I., Santos, Carlos Daza, Francisco Mejia, et al. Multiresistant Extended spectrum betalactamase producing Klebsiella pneumoniae causing an outbreak of nosocomial bloodstream infections. Inf. Cont and Hosp. Epi., 22: 723-725.

Barr, J.G. 1977. Klebsiella: Taxonomy, nomenclature and communication. J. Clin. Path., 30: 943-944.

Barrosa, H., T. Moura, L.M. Lito, A. Duarte, G. Soveral et al. 2000. Survey of Klebsiella pneumoniae producing Extended spectrum betalactamases at a Portugese hospital:TEM-10 as the endemic enzyme. J. Antimicrobial Chemother., 45: 611-616.

Bradley, D., Jett, Thomas, C., Bailley, David, J., Ritchie, Daniel, F., Sahm, et al. 1995. invitro activity of various betalactam antimicrobial agents against clinical isolates of Klebsiella species and Escherichia coli resistant to oxyimino cephalosporins. Antimicrob. Agents Chemother., 39: 1187-1190.

Carmen Pena, Miquel Pujol, Carmen Ardanuy, Francisco Gudiol, Javier Ariza, et al. 1998. Epidemiology and successful control of a large outbreak due to Klebsiella pneumoniae producing Extended spectrum betalactamase Antimicrob agents Chemothe., 42: 53-587.
Chesley Richards, William R. Jarvis, Yolanda Caicrdo, Juan Alonso-Echanove, et al. 2004. Klebsiella pneumoniae blood stream infections among neonates in a high risk nursery in Cali, Colombia. Inf. Cont and Hosp. Epi., 25: 221-225.

Collee, J.G. 1996. Tests for identification of bacteria in Mackie and McCartney, practical medical microbiology 14the ed. Churchill livingstone, 131-149.

Damle, A.S., Anvikar, A.R., R.P. Karyakarte, Malik, A.K. et al. 1999. A one year Prospective study of 3280 surgical wounds Indian J. Med. Microbiol., 17: 129-132.

David, L., Paterson and Robert, A., Bonomo. 2005. Extended spectrum betalactamases: A clinical update. Clin. Microbiol. Rev., 18(4): 657-686

David, L., Paterson, Wen-Chien Ko, Victor, L., $\mathrm{Yu}$, Anne Von Gottberg, et al. 2003. Antibiotic therapy for Klebsiella pneumoniae bacteremia: implications of production of Extended spectrum betalactamases, Clin. Infect. Dis., 39: 23-7.

David, L., Paterson, Wen-Chien Ko, Victor, L., $\mathrm{Yu}$, L.B. Rice et al. 2004. International prospective study of Klebsiella pneumoniae bacteremia: implications of production of extended spectrum betalactamases production in nosocomial infections. Ann. Int. Med., 140: 26-32.

Dennis, S., Hansen, Hazel, M., Aucken, Titi Abiola and Rainer Podschun. 2004. Recommended test panel for differentiation of Klebsiella species on the basis of a trilateral ainetrlaboratory evaluation of 18 biochemical tests. J. Clin. Microbiol., 42: 3665-3669.

Eddy Vercauteren, Patrick Descheemaeker, Margarethaieven, et al. 1997. Comparison of screening methods for detectionof Extended spectrum betalactamases and their prevalence among blood isolates of Escherichia coli and Klebsiella species in a Belgian teaching Hospital, J. Clin. Microbiol., 27: 1421-1428.

Ellie, J.C., Goldstein, Robert, P., Lewis, William, J., Martin et al. 1978. Infections caused by Klebsiella ozaenae: a changing disease spectrum, J. Clin. Microbiol., 8: 413-418. 
Elmer, W., Koneman, Stephen, D., Allen, William M.J. Anda et al. 2006. The Enterobacteriaceae in color atlas of diagnostic microbiology $6^{\text {th }}$ ed. Lippincott, 211-264.

Emily, P., Hyle, Adam, D., Lipworth, Neil, O. Fishman, et al. 2005. Risk factors for increasing multidrug resistance among Extended spectrum betalactamases producing Escherichia coli and Klebsiella species, Clin. Infect. Dis., 40: 1317-24.

Farmer, J.J., Betty, R., Davis, G.K. Morris, F.W. Hickman-Brenner, et al. 1985. Biochemical identification of new species and biogroups of Enterobacteriaceae isolated from clinical specimens. J. Clin. Microbiol., 21: 46-76.

Faustine Ndugulile, Willy Urassa, Nina Langeland, Roland Jureen and Stig Harthug. 2005. Extended spectrum betalactamases among gram negative bacteria of nosocomial origin from a $n$ intensive care unit in a tertiary health facility in Tanzania. BMC Infect. Dis., 5: 86.

Fernandez-Rodrigues, A., Canton, R., Perez-Diaz, J.C., Martinez-Beltran, J., et al. 1992. Aminoglycoside-modifying enzymes in clinical isolates harbouring Extended spectrum betalactamases. Antimicrobial Agents Chemother., 6: 2538-2563.

Freney, J., F. Gavini, D. Izard, H. Alexandre et al. 1986. Nosocomial infection and colonisation by Klebsiella trevisanii. J. Clin. Microbiol., 23: 948-950.

Gary, L., French and Ian Phillips. Antimicrobial resistance in hospital flora. Inf.Cont and Hosp.Epi.: $2^{\text {nd }}$ edi. Edited by C.Glen Mayhall Lippincott Williams and Wilkins 1243-1266.

George, A., Jacoby and Paula Han. 1996. Detection of Extended spectrum betalactamases in clinical isolates of Klebsiella pneumoniae and Escherichia coli. J. Clin. Microbiol., 34: 908-911.

Gioia, S., Babini and David, M. 2000. Livermore. Antimicrobial resistance amongst Klebsiella spp. Collected from intensive care units in southern and western Europe in 1997-1998. J. Antimicrobial. Chemother., 45: 183-189.
Gregory bisson, Neil, O., Fishman, Jean Baldus Patel, Paul, H., Edelstein and Ebbing Lautenbach. Extended spectrum betalactamases producing Klebsiella spp and Escherichia coli: Risk factors for colonization and impact of antimicrobial formulary interventions on colonization prevalence. Inf. Cont and Hosp. Epi., 23: 254-260

Guillermo Saurina, John, M., Quale, Vivek, M., Manikal, David Landman, et al. 2000. Antimicrobial resistance in Enterobacteriaceae in Brooklyn, NY: epidemiology and relation to antibiotic usage pattern. J. Antimicrobial Chemother., 45: 895-898.

Hakki Bahar and Huseyin Tash. Maolecular characterization of TEM- and SHVderived Extended spectrum betalactamases in hospital based Enterobacteriaceae in Turkey. J. Infect. Dis., 58: 162-167.

Hans Jurgen Woske, Thomas Roding, Ines Schulz, Hartmut Lode. 2001. Ventilator associated pneumonia in a surgical intensive care unit: epidemiology, etiology and comparison of three bronchoscopic methods for microbiological specimen sampling. Critical Care, 5: 167-173.

Harish, B.N., Basavaraj, M., Kerur, B. Vishnu Bhat, S. Habeebullah, Uday kumar. 2006. Maternal genital bacteria and colonisation in early neonatal sepsis, Indian $J$. Paediatr., 73: 29-32.

Hernandez, T.M. Coque, A. Pascual, L. MartinezMartinez, R. 2005. Canton and the Spanish group for Nosocomial infections. Nationwide study of Klebsiella pneumoniae and Escherichia coli producing Extended spectrum betalactamases in Spain. Antimicrob agents Chemother., 49: 2122-2125.

Hyunjoo Pai, Cheol-In kang,Kang-Won Choe, KiDoek Lee, et al. 2004. Epidemiology and clinical features of bloodstream infections caused by Amp C type beta lactamases producing Klebsiella pneumoniae. Antimicrobial Agents Chemother., 48: 3720-3728.

Ingo Stock and Bernd Wiedemann. 2000. Natural antibiotic susceptibility of Klebsiella pneumoniae, $K$. oxytoca, K.planticola, 
K.ornitholytica and K. terrigena strains. J. Med. Microbiol., 50: 396-406.

Janis Weiner, John, P., Quinn, Karen Bush, Patricia, A., Bradford, et al. 1998. Multiple antibiotic resistant Klebsiella and Escherichia coli in nursing homes. JAMA, 281: 517-523.

Jen Hsein Wang, Yung-Ching Liy, Muh-Yong Yen, Susan Shin-Jung Lee et al. 1998. Liver abscess due to Klebsiella pneumoniae in Taiwan. Clin. Infec. Dis., 26: $1434-8$.

Jenny, S., Carter, Francis, J., Bowden, Ivan Bastian, Garry, M., Myers et al. 1999. Phylogenetic evidence for reclassification of Calymmatobacterium granulomatis as Klebsiella granulomatis comb.nov. Int. J. Syst. and Evol. Microbiol., 49: 1695-1700.

Livermore, D.M. and M. Yuan. Antibiotic resistance and production of Extended spectrum betalactamase amongst Klebsiella species from intensive care units in Europe. J. Antimicrob. Chemother., 38: 409-424.

Mackenzie, F.M., S.G.B. Amyes, K.J. Forbes, T. Dorai John, et al. 1986. Emergence of carbapenem resistant Klebsiella pneumoniae. Lancet, 783.

Malik, A., Se Hussain, M. Shahid, H.M. Khan, A.J. Ahmed. 2003. Nosocomial Klebsiella infections in neonates in a tertiary care hospital, Indian J. Med. Microbiol., 21: 113:2:82-86

Monnet, D., and J. Freney. 1994. Method for differentiating Klebsiella planticola and
Klebsiella terrigena from other Klebsiella species. J. Clin. Microbiol., 32: 1121-1122.

Rodrigues, C., P. Joshi, S.H. Jain, M. Alphonse et al. 2004. Detection of betalactamases in nosocomial gram negative clinical isolates, Indian J. Med. Microbiol., 22: 247-250.

Shukla, I., R. Tiwari, M. Agarwal. 2004. Prevalence of Extended spectrum betalactamases producing Klebsiella pneumoniae in a tertiary care hospital. Indian J. Med. Microbiol., 22: 87-91.

Subha, A., Ananthan, S. 2002. Extended spectrum betalactamase mediated resistance to third generation cephalosporins among Klebsiella pneumoniae in Chennai. Indian J. Med. Microbiol., 20(2): 92-95.

Subha, A., S. Ananthan, S.V. Alavandhi. 2001. Extended spectrum betalactamase production and multidrug resistance in Klebsiella species isolated from children under five with intestinal and extraintestinal infections, Indian J. Med. Res., 113: 181-185.

Subha, A., S. Ananthan. 2005. Cefoxitin resistance mediated by a loss of a porin in clinical strains of Klebsiella pneumoniae and Escherichia coli., Indian J. Med. Microbiol., 23(1): 20-23.

Subha, A., V. Renuka Devi, S. Ananthan. 2003. Amp $\mathrm{C}$ betalactamase producing multidrug resistant strains of Klebsiella species and Escherichia coli isolated from children under five in Chennai, Indian J. Med. Res., 117: 13-18.

\section{How to cite this article:}

Sangamithra, V., Shamsadh, Kalyani and Mallika. 2017. Prevalence of Extended Spectrum Beta-Lactamase Producing Klebsiella Species in an Intensive Care Unit. Int.J.Curr.Microbiol.App.Sci. 6(5): 1449-1470. doi: https://doi.org/10.20546/ijcmas.2017.605.159 